\title{
Positron Annihilation in Defected Monocrystalline Gold Samples
}

\author{
J. PAjąk, W. Rudzińska, M. Szuszkiewicz \\ and M. GarlowsKa \\ Institute of Physics, Opole University, Oleska 48, 45-052 Opole, Poland \\ Angular distribution of the positron annihilation quanta was measured \\ for monocrystalline gold samples, oriented in (110) and (111) directions. The \\ samples were deformed by elongation for different deformation degrees. The \\ $S$ and $W$ parameters as a function of deformation degree of the sample were \\ determined. It was found that the dynamics of the dislocations and vacancy \\ generation during the sliding of some crystallographic planes, depends on \\ the crystallographic direction.
}

PACS numbers: $78.70 . \mathrm{Bj}$

\section{Introduction}

It is well known that metals under the action of the external forces are deformed, first elastically, and after exceeding elasticity limit, the deformation starts to be plastic. Plastic deformation requires the dislocation movement by translation in the slip plane or by climbing [1]. During the climbing process the edge (or mixed) dislocations are moving by adjoining or emitting vacancies to or from the extra half plane. This way the dislocations are the sources (or sinks) of vacancies. The slip starts when the tangential stress in the easy slip plane and easy slip direction (close packed planes and directions) caused by external forces, reaches some threshold value - so-called critical tangential stress $\left(\tau_{\mathrm{cr}}\right)$. The dependence of the tangential stress $\tau$ in the slip direction on the elongation force $F$ is presented by the relation $[2,3]$ :

$$
\tau=\frac{F}{S_{0}} \cos \lambda \cos \phi
$$

where $F$ - the elongation force, $S_{0}$ - the cross-sectional area of the elongated sample, $\lambda$ - the angle between slip direction and force $F, \phi$ - the angle between $S_{0}$ plane and slip the plane. 
The value of $\tau$ depends on the orientation of monocrystal in relation to the force $F$. The $\tau$ attains maximum when the angle $\phi$ between the sample axis and the slip plane and slip direction is $45^{\circ}$. For $\phi=90^{\circ}$ or $0^{\circ}, \tau$ is 0 . In the case of gold the easy slip planes are $\{111\}$ planes and easy slip directions are (110) directions. The processes occurring during the plastic deformation are studied mainly by microscopic techniques. It is well known that during the dislocation movement the vacancies are created due to the sliding of some crystallographic planes. The possibilities to observe the behaviour of these vacancies by the microscopic techniques are rather limited. This aspect of mechanical deformation can be, however, very effectively studied by the positron annihilation methods. In paper [4], the angular correlation of positron annihilation quanta (ACPAQ) method was used for investigation of pure and hydrogenated palladium samples deformed by uniaxial tension. It was found that after elongation, in both the pure and hydrogenated palladium samples, the positrons are trapped in defects larger than vacancy (vacancy clusters and similar defects) depending on the elongation degree of the sample. In paper [5] the ACPAQ method and lifetime measurements were used for investigation of the polycrystalline silver samples deformed by elongation. The results indicated the dominant role of vacancies and vacancy clusters created during the deformation process. In paper [3] the ACPAQ method was used for investigation of the monocrystalline silver samples oriented in [110] and [111] direction. From these investigations we concluded that the dynamics of the dislocations and vacancy generation during the sliding of some crystallographic planes depends on the crystallographic direction. The aim of this paper is to apply the ACPAQ method for investigation of changes in the annihilation parameters caused by uniaxial elongation of the monocrystalline gold samples oriented in the [110] and [111] directions.

\section{Experimental}

Monocrystalline gold samples oriented in the [110] and [111] directions, of the $4 \mathrm{~N}$ purity, with dimensions at $15 \times 10 \times 1 \mathrm{~mm}^{3}$ used in this experiment, were tempered for four hours at $980 \mathrm{~K}$ and slowly cooled to room temperature. After that, one of the samples was placed in a specially prepared setup, by means of which the sample was elongated to different relative elongations. For each elongation value the ACPAQ curve was measured using the standard correlation spectrometer with a long slit geometry.

\section{Results and discussion}

Angular correlation curves of positron annihilation quanta have been measured for monocrystalline gold samples, oriented in (110) and (111) directions, submitted to increasing relative static uniaxial elongation by: $0.00,0.20,0.40$, $0.60,0.80,1.20,1.40,1.60,1.80,2.00,2.40,2.80,3.20,4.80,5.60,7.20,8.00$, $9.00,10.00,11.00,12.00,14.00$, and $15.00 \%$. 
For presentation of the changes in the ACPAQ curves, caused by the sample elongation, the $S$ and $W$ parameters, defined as ratio of the area under the ACPAQ curve confined in the interval of angles ranging from $-4 \mathrm{mrad}$ to $+4 \mathrm{mrad}$ and the sum of the areas ranging from $-12 \mathrm{mrad}$ to $-8 \mathrm{mrad}$ and from $+8 \mathrm{mrad}$ to $+12 \mathrm{mrad}$, respectively, to the area under the whole ACPAQ curve, have been calculated for each of the ACPAQ curves. The dependences of the $S$ parameter on the relative elongation of the monocrystalline gold samples, oriented in the (110) and (111) directions are presented in Fig. 1. As it was given in paper [3], the orientation of the sample in relation to tensile force means that the tensile force is parallel to plane that is perpendicular to given direction. In the case of our experiment they are $\{110\}$ and $\{111\}$ planes.

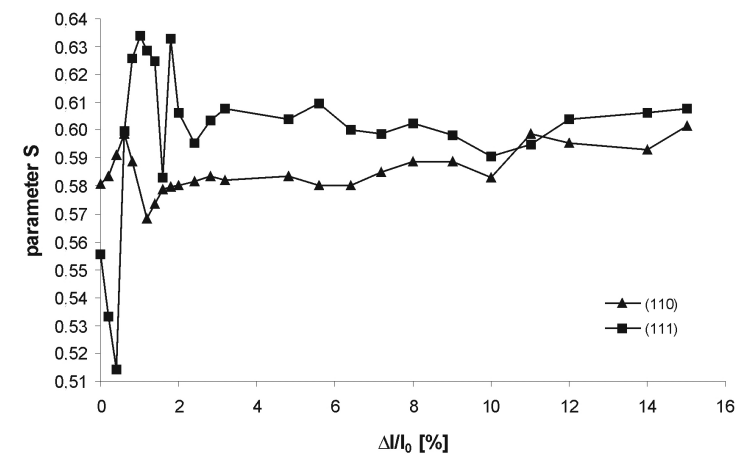

Fig. 1. The dependence of the $S$ parameter on the relative elongations of the samples oriented in (110) and (111) directions.

In the case of sample oriented in (110) direction, the angle between the easy slip plane (i.e. the $\{111\}$ plane) and tensile force is $45^{\circ}$. In agreement with formula (1), in order to reach the critical value of the tangential stress and causing the consecutive slides, one needs to use the lowest value of the tensile force. The internal stress in the sample increases before every next slip. As a result of increase in the internal stress, dislocation and vacancy-type defects are formed. After reaching the critical value of the tangential stress, the slip is activated in $\{111\}$ planes. After finishing the slip, the sample is easing of the tension partly, and densities of the dislocations and vacancies are decreased. The jump-like character of the process of set going of slip on easy slip plane is visualized by characteristic consecutive increases and decreases in the course of $S$ parameter as a function of elongation of the sample, which is presented in Fig. 1. In the case of the sample oriented in (111) direction, the tensile force is parallel to the $\{111\}$ plane. In agreement with formula (1), the tangential stress in the easy slip plane, that is $\{111\}$ plane equals 0 . The slip can occur in the other planes, for example $\{110\}$ plane, which is not easy slip plane, and requires larger value of the critical tangential stress to activate the slip. The dependence of the $S$ parameter value on 
the elongation of sample oriented in (111) direction is shown in Fig. 1 (marked by black squares). Because of the reasons mentioned above, the changes in the course of $S$ parameter for sample oriented in (111) direction are much larger than those for sample oriented in (110) direction, and the peaks occur at the largest values of the relative elongations.

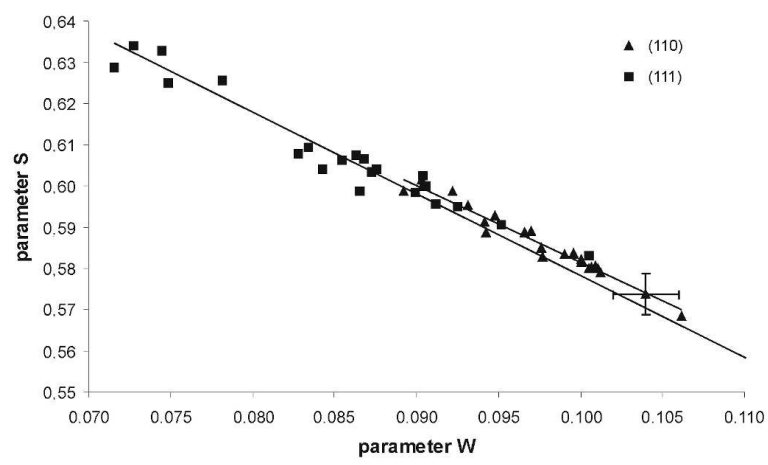

Fig. 2. The dependence of the $S$ parameter on the relative elongations of the samples oriented in (110) and (111) directions.

The dependence of the $S$ parameter on the $W$ parameter is presented in Fig. 2. For all the investigated monocrystalline gold samples the experimental data points are located along straight lines sloped under some angle to the $W$ coordinate. The slope of these lines gives the value of the $R_{\mathrm{D}}$ parameter, characteristic of the type of defects trapping the positrons in the investigated samples [6]. In the case of investigated samples these traps are, most probably, dislocations and vacancies generated during the plastic deformation by elongation, which corroborates the dislocation model of plastic deformation.

\section{References}

[1] R.W.K. Honeycombe, The Plastic Deformation of Metals, Edward Arnold, Cambridge 1968.

[2] M. Głowacka, Metallography, Politechnika Gdańska, Gdańsk 1966 (in Polish).

[3] J. Pajạk, W. Rudzińska, Cz. Szymański, S. Ga̧sior, K. Malisz, in: Proc. 34th Polish Seminar on Positron Annihilation, Turawa (Poland) 2002, Ed. K. Jerie, Institute of Physics, University of Opole, Institute of Experimental Physics, University of Wrocław, Opole 2002, p. 55.

[4] W. Rudzińska, J. Paja̧k, Cz. Szymański, R. Szatanik, Acta Phys. Pol. A 99, 479 (2001).

[5] J. Pająk, W. Rudzińska, R. Pietrzak, Cz. Szymański, W. Smiatek, Phys. Scr. XX, 2 (2003).

[6] W. Triftshäuser, Phys. Rev. B 12, 4634 (1975). 